Proof. Let $X_{1}$ be the set of all $x$ in $X$ for which $|x|=1$. Since each $G_{j} \subseteq G=\operatorname{gp} X$, we conclude by Lemma 8 that each $G_{j} \subseteq$ gp $X_{1}$, and hence that $G=\operatorname{gp} X_{1}$. It follows from the irreducibility of $X$ that $X=X_{1}$.

This completes the proof of Grushko's Theorem.

\title{
BIBLIOGRAPHY
} 182.

1. I. A. Grushko, On the bases of a free product of groups, Mat. Sb. 8 (1940), 169-

2. A. G. Kurosh, The theory of groups. II, Chelsea, New York, 1955.

3. R. C. Lyndon, Length functions in groups, Math. Scand. 12 (1963), 209-234.

4. B. H. Neumann, On the number of generators of a free product, J. London Math. Soc. 18 (1943), 12-20.

UNIVERSITY OF MICHIGAN

\section{GENERALIZED FUNCTIONS OF SYMMETRIC MATRICES}

\section{MARVIN MARCUS ${ }^{1}$ AND MORRIS NEWMAN}

1. Introduction. In an abstract published in 1961 [4] we announced the following result:

Let $A$ be an n-square positive semi-definite matrix and assume that $A \geqq S$ where $S$ is doubly stochastic. Then

$$
\operatorname{per}(A) \geqq n ! / n^{n} \text {. }
$$

The notation $A \geqq S$ means $a_{i j} \geqq s_{i j}, i, j=1, \cdots, n$. A doubly stochastic (d.s.) matrix has non-negative entries and every row and column sum is 1 . The permanent, per $(A)$, is the function defined by

$$
\operatorname{per}(A)=\sum_{\sigma \in \mathcal{S}_{n}} \prod_{i=1}^{n} a_{i \sigma(i)}
$$

where the summation extends over the whole symmetric group of degree $n, S_{n}$.

In 1962 [3] we also proved that:

If $S$ is an $n$-square positive semi-definite symmetric matrix which is doubly stochastic in the extended sense then

$$
\operatorname{per}(S) \geqq n ! / n^{n} .
$$

Received by the editors June 8,1964 .

${ }_{1}^{1}$ The work of this author was supported by NSF Grant GP-1085. 
A matrix is d.s. in the extended sense if every row and column sum is 1 ; however, the elements need not all be non-negative. The inequality (1.3) constitutes what is currently known about a conjecture of van der Waerden that states:

$$
\operatorname{per}(S) \geqq n ! / n^{n}
$$

for any $n$-square d.s. matrix $S$.

Observe that for the inequality (1.1) no assumption is made about $S$ being positive semi-definite, otherwise we obviously could get the result from (1.3). One might wonder that if a d.s. matrix $S$ exists for which $A \geqq S$, then perhaps a positive semi-definite matrix $S_{1}$ exists which is d.s. in the extended sense and satisfies $A \geqq S_{1}$. In other words one could hope to relax the d.s. condition in exchange for positive semi-definiteness. This is unfortunately not true. For, take

$$
A=\left(\begin{array}{ccc}
\frac{1}{3} & 0 & \frac{3}{4} \\
0 & 1 & 0 \\
\frac{3}{4} & 0 & 3
\end{array}\right)
$$

which is obviously positive semi-definite. Clearly $A \geqq S$ where

$$
S=\left(\begin{array}{ccc}
\frac{1}{4} & 0 & \frac{3}{4} \\
0 & 1 & 0 \\
\frac{3}{4} & 0 & \frac{1}{4}
\end{array}\right)
$$

$S$ is not positive semi-definite. Thus we can try for an $S_{1}$ such that $A \geqq S_{1}, S_{1}$ is d.s. in the extended sense, and $S_{1}$ is positive semi-definite. Set

$$
S_{1}=\left(\begin{array}{ccc}
a & c & 1-(a+c) \\
c & b & 1-(b+c) \\
1-(a+c) & 1-(b+c) & a+b+2 c-1
\end{array}\right) .
$$

If $S_{1}$ is to be positive semi-definite then

$$
0 \leqq a(a+b+2 c-1)-(1-a-c)^{2},
$$

which simplifies to

$$
(b+1) a \geqq(c-1)^{2} .
$$

If $A \geqq S_{1}$ then $a b+a \leqq \frac{2}{3}$ and $c \leqq 0$, which are incompatible with (1.4).

Since the appearance of the original abstract we have done a substantial amount of work on generalized matrix functions as originally 
defined by I. Schur [7]. Thus let $G$ be a subgroup of $S_{n}$ and let $\chi$ be a character (of arbitrary degree) of $G$. Following Schur we define the generalized matrix function $d_{x}$ by

$$
d_{\chi}(A)=\sum_{\sigma \in G} \chi(\sigma) \prod_{i=1}^{n} a_{i \sigma(i)}
$$

for any $n$-square matrix $A$. Clearly if $G=S_{n}$ and $\chi$ is the character of $G$ identically 1 then $d_{x}(A)=\operatorname{per}(A)$. Schur [7, Theorem 1] has shown that $d_{\chi}(A)>0$ for positive definite hermitian $A$. It follows that $d_{\chi}(A)$ $\geqq 0$ for positive semi-definite $A$. The purpose of this paper is to prove the following:

THEOREM. Let $A$ be an $n$-square positive semi-definite real symmetric matrix and assume that $A \geqq S$ where $S$ is doubly stochastic. Then

$$
d_{\chi}(A) \geqq m(\chi) / n^{n}
$$

where

$$
m(\chi)=\sum_{\sigma \in G} \chi(\sigma)
$$

For $G=S_{n}$ and $\chi \equiv 1, m=m(\chi)=n !$, and (1.6) specializes to (1.1). It is always true of course that $m(\chi)$ is either 0 or a positive integral multiple of the order of $G$. More precisely let $\chi=\chi_{1}+\cdots+\chi_{k}$ be a representation of the character $\chi$ as a sum of irreducible characters. Then $m(\chi) / g$ is the number of $\chi_{i}$ in this representation which equal the trivial character 1 . Here $g$ is the order of $G$. Thus for nontrivial irreducible $\chi$ our theorem reduces to Schur's result.

2. Preliminary results. The proof of the inequality (1.6) depends on three theorems of interest in themselves.

THEOREM 1. If $V$ and $W$ are arbitrary $n$-square complex matrices then

$$
\left|d_{x}(V W)\right|^{2} \leqq d_{\chi}\left(V V^{*}\right) d_{x}\left(W^{*} W\right)
$$

This result has appeared as a research announcement [2].

Theorem 2. If $A$ is a symmetric $n$-square matrix and $A>$ ) (i.e., $\left.a_{i j}>0, i, j=1, \cdots, n\right)$ then there exists a unique diagonal matrix $D=\operatorname{diag}\left(d_{1}, \cdots, d_{n}\right), d_{i}>0, i=1, \cdots, n$, such that $D A D$ is d.s.

The authors knew this theorem at the time of the announcement [4] and shortly thereafter a constructive proof was found by Maxfield and 
Minc [6]. Independently Sinkhorn [8], [9] proved a closely related result.

Theorem 3. Let $D$ be the diagonal matrix described in Theorem 2 . Moreover, assume that $A \geqq S$ where $S$ is d.s. Then

$$
\prod_{i=1}^{n} d_{i} \leqq 1 .
$$

Proof. For any $n$-square matrix $X$ let $r_{i}(X)$ denote the $i$ th row sum of $X$. Now $A \geqq S$ implies that $D A D \geqq D S D$ and since $D A D$ is d.s. we have

$$
1=r_{i}(D A D) \geqq r_{i}(D S D)=d_{i} \sum_{j=1}^{n} s_{i j} d_{j}
$$

Thus

$$
1 \geqq \prod_{i=1}^{n} d_{i} \prod_{i=1}^{n} \sum_{j=1}^{n} s_{i j} d_{j}
$$

Let

$$
g(X)=\prod_{i=1}^{n}\left(\sum_{j=1}^{n} x_{i j} d_{j}\right)^{1 / n}
$$

where $X$ is an arbitrary d.s. matrix. It is a well-known result of Birkhoff [1] that the totality $\Omega_{n}$ of $n$-square d.s. matrices is a convex polyhedron with the permutation matrices as vertices. If $X, Y \in \Omega_{n}$, $0 \leqq \theta \leqq 1$, then the Hölder inequality implies that

$$
\begin{aligned}
g(\theta X+(1-\theta) Y) & =\prod_{i=1}^{n}\left(\sum_{j=1}^{n}\left(\theta x_{i j}+(1-\theta) y_{i j}\right) d_{j}\right)^{1 / n} \\
& =\prod_{i=1}^{n}\left(\theta \sum_{j=1}^{n} x_{i j} d_{j}+(1-\theta) \sum_{j=1}^{n} y_{i j} d_{j}\right)^{1 / n} \\
& \geqq \theta g(X)+(1-\theta) g(Y) .
\end{aligned}
$$

Hence $g$ is concave on $\Omega_{n}$ and assumes its minimum on a permutation matrix. The value of $g$ on any permutation matrix is just $\left(\prod_{i=1}^{n} d_{i}\right)^{1 / n}$. From (2.3) we can conclude that

$$
1 \geqq \prod_{i=1}^{n} d_{i}(g(X))^{n} \geqq \prod_{-}^{n} d_{i}^{2}
$$

and (2.2) follows. 
3. Proof of the Theorem. In [5] it was proved that if $R$ is any d.s. positive semi-definite matrix then the positive semi-definite determination of the square root of $R, R^{1 / 2}$, is d.s. in the extended sense. In Theorem 1 let $V=R^{1 / 2}$ and $W=J_{n}$, the matrix with every entry $1 / n$. Then $R^{1 / 2} J_{n}=J_{n}, J_{n}^{2}=J_{n}$, and thus

$$
\left|d_{\chi}\left(J_{n}\right)\right|^{2} \leqq d_{\chi}(R) d_{\chi}\left(J_{n}\right) \text {. }
$$

It is known from Schur's theorems on the $d_{x}$ function that $d_{x}(R) \geqq 0$. ( $R$ is positive semi-definite.) Hence whether $m(\chi)$ is 0 or not we have

$$
d_{\chi}(R) \geqq d_{\chi}\left(J_{n}\right)=m(\chi) / n^{n} .
$$

We can assume by continuity that $A>0$ in proving (1.6). By Theorem 2 choose a diagonal matrix $D=\operatorname{diag}\left(d_{1}, \cdots, d_{n}\right), d_{i}>0$, $i=1, \cdots, n$ for which $D A D=R$ is d.s. Then

$$
d_{\chi}(D A D)=d_{\chi}(R) \geqq m(\chi) / n^{n} .
$$

But by Theorem 3

$$
d_{x}(D A D)=\prod_{i=1}^{n} d_{i}^{2} d_{x}(A) \leqq d_{x}(A)
$$

The inequalities (3.2) and (3.3) complete the proof.

\section{REFERENCES}

1. G. Birkhoff, Tres observaciones sobre el algebra lineal, Univ. Nac. Tucumán Rev. Ser. A 5 (1946), 147-150.

2. Marvin Marcus, On two classical theorems of I. Schur, Bull. Amer. Math Soc. 70 (1964), 685-688.

3. Marvin Marcus and Morris Newman, Inequalities for the permanent function, Ann. of Math. 75 (1962), 47-62.

4. - The permanent of a symmetric matrix, Abstract 587-85, Notices Amer. Math. Soc. 8 (1961), 595.

5. Marvin Marcus and Henryk Minc, Some results on doubly stochastic matrices, Proc. Amer. Math. Soc. 76 (1962), 571-579.

6. John Maxfield and Henryk Minc, $A$ doubly stochastic matrix equivalent to a given matrix, Notices Amer. Math. Soc. 9 (1962), 309.

7. I. Schur, Über endliche Gruppen und Hermitesche Formen, Math. Z. 1 (1918), 184-207.

8. Richard Sinkhorn, On best doubly stochastic estimates, Notices Amer. Math. Soc. 9 (1962), 294.

9. - A relationship between arbitrary positive matrices and doubly stochastic matrices, Ann. Math. Statist. 35 (1964), 867-879.

University of California, Santa Barbara AND National Bureau of Standards, Washington, D. C. 